\title{
In-Fiber Fractional Signal Processing: Recent Results and Applications
}

\author{
Christian Cuadrado-Laborde ${ }^{* \dagger+}$, Luis Poveda-Wong*, Antonio Carrascosa*, José Luis Cruz ${ }^{*}$, \\ Antonio Díez , Miguel V. Andrés", Member, IEEE \\ *ICMUV, Universidad de Valencia, Burjassot, Spain \\ Tel: +34 963543338, e-mail: miguel.andres@uv.es \\ ${ }^{\dagger}$ Instituto de Física Rosario (CONICET-UNR), Rosario, Argentina \\ ${ }^{\ddagger}$ Pontificia Universidad Católica Argentina, Facultad de Química e Ingeniería, Rosario, Argentina
}

\begin{abstract}
The implementation of mathematical operators using photonic signal processing -as for example, conventional differentiators and integrators - is particularly well suited to overcome the speed and bandwidth limitations of electronics. In the Laboratory of Fiber Optics of the University of Valencia we work on the development of infiber time-domain fractional operators and their applications. In the last years we have made some specific proposals to perform photonic fractional differentiation (PFD), photonic fractional integration (PFI), photonic fractional Hilbert transform (PFHT), and photonic fractional Fourier transform (PFFT), using fiber-based technologies. Recently, we have been able to implement experimentally some of these theoretical proposals, and we have found specific applications for the $0.5^{\text {th }}$-order differentiation and the photonic fractional Fourier transform in the area of phase recovery of optical pulses.
\end{abstract}

Keywords: photonic signal processing, optical fiber technologies, fractional operators, phase recovery, instantaneous frequency.

\section{INTRODUCTION}

Signal processing in optical networks based on optical-to-electrical conversion, and back electrical-to-optical conversion, is limited by the electronic sampling rate. Photonic signal processing can overcome this limitation, working directly in the optical domain. A photonic signal processor has the ability to perform a certain operation directly on the complex field envelope of a given light signal. In our case, we have focused our attention on analogue processing using optical fiber technologies [1].

First, integer operators -as for example, conventional differentiators [2], integrators [3], and Fourier transformers [4] - were investigated and a number of proposals have been reported. In the Laboratory of Fiber Optics of the University of Valencia we work on the development of in-fiber time-domain fractional operators and their applications. Since fractional operations play an important role in information processing, we started a research line to translate to the photonic domain the flexibility provided by fractional mathematical operations.

For years, we were making some theoretical proposals - to the best of our knowledge, we were the first to propose a photonic fractional differentiator [5], and a photonic fractional integrator [6] - and more recently we have been working on the experimental implementation of fractional differentiators [7, 8], and fractional Fourier Transformers [9]. Moreover, we have found specific applications for the $0.5^{\text {th }}$-order differentiation and the photonic fractional Fourier transform in the area of phase recovery of optical pulses [10, 11].

Here, we will focus on this last part of our work, the experimental implementations of photonic operators with specific application in the area of phase recovery (i. e., instantaneous frequency) of optical pulses.

\section{FRACTIONAL DIFFERENTIATION USING LONG PERIOD GRATINGS}

It is well known that the process of fractional time differentiation, $\mathrm{d}^{n} f(t) / \mathrm{d} t^{n}$, is equivalent to a filtering action in the spectral domain performed by an ideal filter with the transfer function $H_{n}(v)=(j 2 \pi v)^{n}$, where $j=\sqrt{ }-1, v$ is the baseband frequency $\left(v=\left(\omega-\omega_{0}\right) / 2 \pi\right.$, being $\omega$ the optical frequency and $\omega_{0}$ the carrier frequency), and $n \in \mathfrak{R}^{+}$is the fractional order. The transfer function of this filter has a $n \times \pi$ phase discontinuity at $v=0$ and a $|v|^{n}$ dependence for the amplitude. In-fiber long period gratings (LPG) can provide this type of transfer function, $H_{n}(v)$, with a reasonable degree of precision [12]. If the target is a specific fractional order $n$, then the length $z$ of the LPG will have to be selected according to the analytical expression $n=1-\left[1-(z / L)^{2}\right]^{1 / 2}$, being $L$ the full coupling length. LPG exhibit the right spectral response for signals $f(t)$ which spectral bandwidth is higher and smaller than certain minimum and maximum values [12]. In our case, since we were interested in picosecond pulses, we needed to develop a technique for the fabrication of narrow band LPG [13].

Figure 1(a) shows the amplitude response of a LPG design to perform a $0.5^{\text {th }}$ order differentiation, i.e. a semidifferentiation, at about $1038 \mathrm{~nm}$, which linewidth is about $1 \mathrm{~nm}$. Figures 1(b) and 1(c) give the amplitude and instantaneous frequency (i. e., $\mathrm{d} \varphi / \mathrm{d} t$ ) obtained after processing a linearly chirped pulse with an hyperbolic secant profile, $g(t)=\operatorname{sech}\left(t / T_{0}\right) \exp \left(j C t^{2} / 2 T_{0}^{2}\right), T_{0}=13 \mathrm{ps}, C=-30$. A good matching between theory and experiment is obtained. The peak that the instantaneous frequency exhibits in the middle of the curve is a characteristic 
signature of the phase discontinuity produced by the differentiator, which is not a discontinuity but a fast phase variation in a short frequency range.

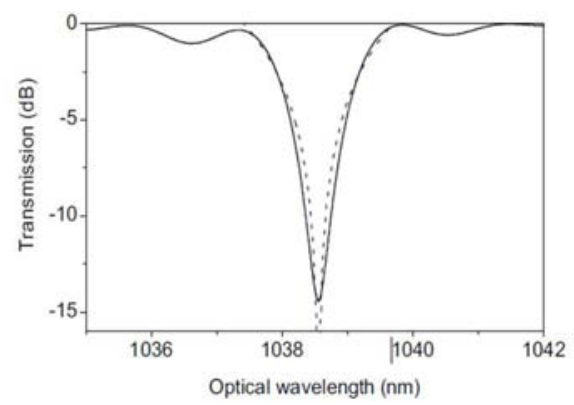

(a)

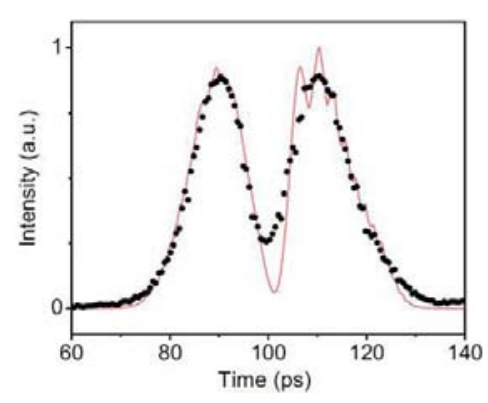

(b)

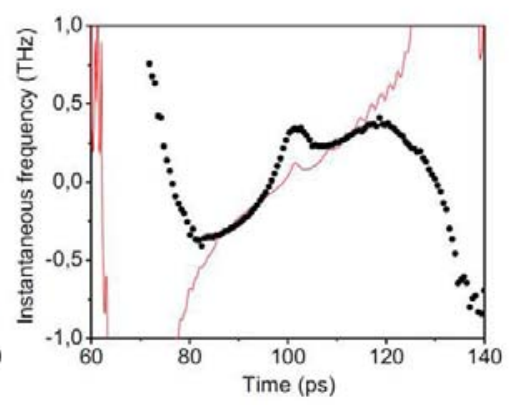

(c)

Figure 1: (a) Measured optical spectrum of the LPG and theoretical intensity response of a $0.5^{\text {th }}$ order fractional differentiator, solid and dashed lines, respectively. Measured temporal intensity profile (b) and instantaneous frequency (c) of the light pulse at the output of the LPG (scatter points), and simulated response of an ideal $0.5^{\text {th }}$ order differentiator (solid curve).

Semi-differentiation is particularly interesting because it enables a simple determination of the instantaneous frequency of a pulse by processing a spectrally shifted signal [10]:

$$
\frac{\mathrm{d} \varphi(t)}{\mathrm{d} t} \approx \frac{\left|\frac{\mathrm{d}^{0.5}}{\mathrm{~d} t^{0.5}}\left[g(t) \exp \left(j \omega_{s} t\right)\right]\right|^{2}}{|g(t)|^{2}}-\omega_{s}
$$

where $\omega_{s}$ is the frequency shift that the techniques requires. Thus, in order to compute the instantaneous frequency of a signal $g(t)$, only two intensity measurements are required, i.e., the numerator and the denominator on eq. (1), but no iterative algorithm is needed. Figure 2 gives the results that were obtained when carrying out the characterization of the same pulse used in Fig. 1(b) and 1(c). The intensity of the input pulse was fitted to a hyperbolic secant profile with $T_{0}=13 \mathrm{ps}$, and the instantaneous frequency values computed with eq. (1) were fitted with a linear chirp parameter $\mathrm{C}=-30$.
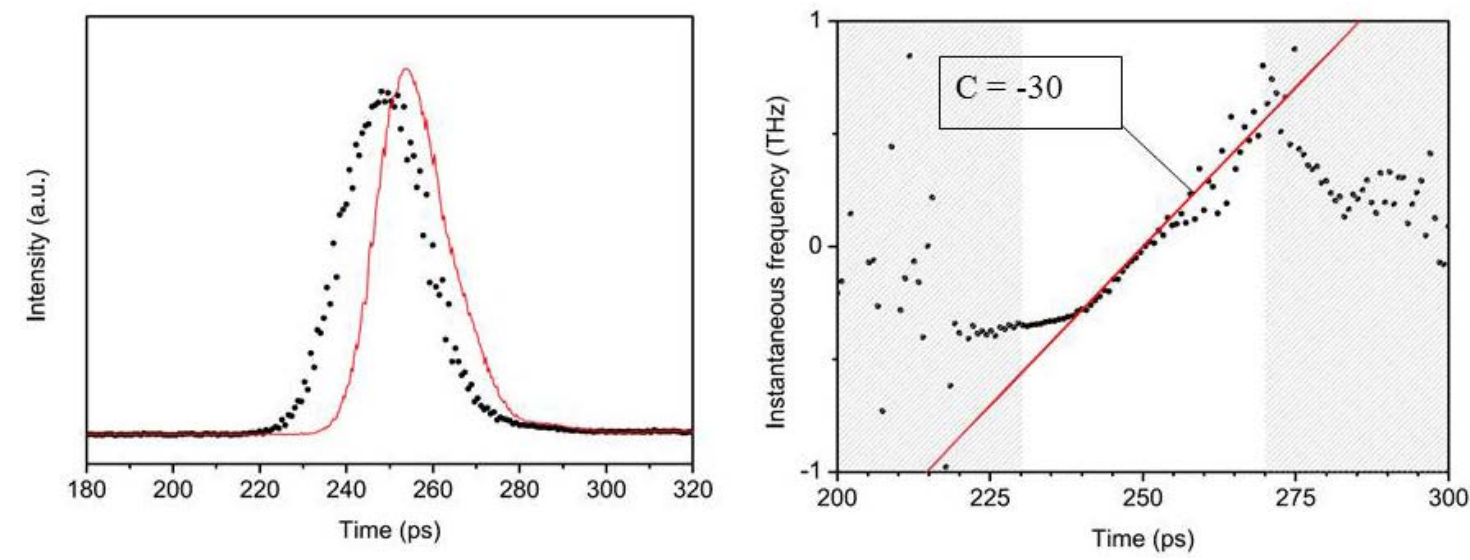

Figure 2: (Left) Temporal intensity profiles of the spectrally shifted optical pulse under test, and $0.5^{\text {th }}$ order fractional differentiation, scatter points and solid line, respectively; (Right) Instantaneous frequency values obtained by means of eq. (1), and linear fit, scatter points and solid curve, respectively.

\section{PHOTONIC FRACTIONAL FOURIER TRANSFORMER}

The Fractional Fourier Transform (FFT) has found widespread use for optical signal processing in the spatial domain, and recently it has been investigated in the time domain. The intensity of the FFT of a pulse $f(t)$ can be obtained experimentally by simple propagation in a spool of optical fiber properly chosen [9]. Additionally, we were able to demonstrate experimentally that the instantaneous frequency can be obtained by using the temporal version of the transport-of-intensity equation:

$$
\frac{\mathrm{d} \varphi(t)}{\mathrm{d} t}=\frac{1}{|f(t)|^{2}}\left[\frac{\partial\left|f_{\alpha}(t)\right|^{2}}{\partial \alpha} * H(t)\right]
$$


where * is the convolution, $H(t)$ is the Heaviside step function, $f_{\alpha}(t)$ is the FFT of $f(t)$, and $\alpha$ is the parameter the defines the FFT order. In order to perform the measurements, the derivative $\partial / \partial \alpha$ will be replaced by a finite difference:

$$
\frac{\partial\left|f_{\alpha}(t)\right|^{2}}{\partial \alpha} \approx \frac{\left|f_{\alpha}(t)\right|^{2}-|f(t)|^{2}}{\alpha}
$$

Thus, by measuring the intensity profiles of the input pulse and its FFT, we can obtain the instantaneous frequency in a single computational step, again avoiding the use of iterative algorithms. The parameter $\alpha$ is determined by the chromatic dispersion $\beta_{20}$ and the length $L$ of the optical fiber $\left(\alpha=\beta_{20} L\right)$. A good experimental implementation requires that $\alpha$ is sufficiently large to reduce the errors of the numerator of eq. (3), but small enough to ensure that the finite difference gives a good numerical evaluation of the derivative.

Figure 3 gives the characterization of a pulse $f(t)$ with a dispersion line of $315 \mathrm{~m}\left(\alpha=7.94 \mathrm{ps}^{2}\right)$, we can see the intensity profile of the input pulse which can be fitted to a hyperbolic secant profile with $T_{0}=12$ ps, the intensity profile of the FFT of the pulse, and the difference corresponding to the numerator of eq. (3). Figure 3 (b) gives the instantaneous frequency computed using eq. (2) (Exp. FrT), the theoretical curve obtained with a parabolic phase that corresponds to a chirp parameter $C=-11$, the phase obtained with eq. (2) using a numerically simulated FFT (Sim. FrT), and the comparison with a standard phase recovery Gerchberg-Saxton algorithm (Exp. GSA). We can observe that our technique is rather robust against noise in comparison with a standard Gerchberg-Saxton algorithm.
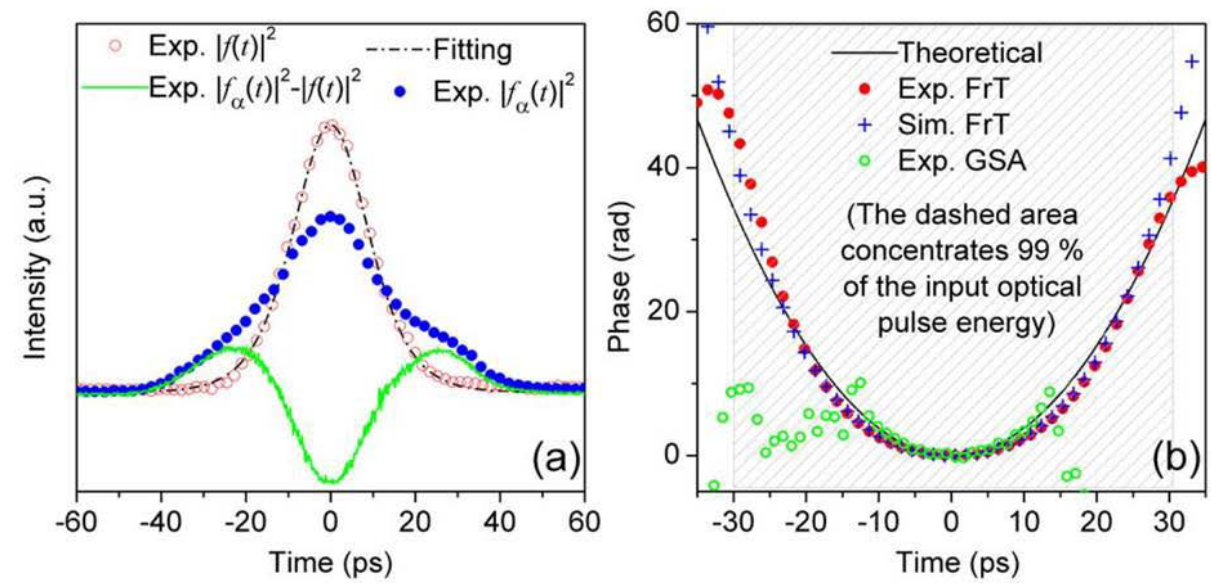

Figure 3: (a) Measured input intensity profile (plus the corresponding fitting), its FFT measured at the output of a fiber spool, and the difference; (b) Phase recovered by the proposed experimental technique (Exp. FrT), and comparison with other approaches.

\section{CONCLUSIONS}

The development of time-domain fractional calculus using photonic signal processing - in addition to the conventional calculus based on normal differentiation and integration - is feasible and can be performed using optical fiber technologies. The experimental implementation on fractional differentiation and fractional Fourier transform using passive fiber devices have pushed forward the development of new applications in the area of phase recovery.

\section{ACKNOWLEDGEMENTS}

The authors acknowledge the Ministerio de Economía y Competitividad (MINECO) of Spain and Fonds Européen de Développement Économique et Régional (FEDER) (Ref.: TEC2016-76664-C2-1-R), as well as the Generalitat Valenciana (Ref.: PROMETEOII/2014/072). C. Cuadrado-Laborde acknowledges the financial support from project PICT 2008-1506 (ANPCyT, Argentina) and PIP 11220150100607CO (CONICET, Argentina).

\section{REFERENCES}

[1] C. Cuadrado-Laborde, L. Poveda-Wong, A. Carrascosa, J. L. Cruz, A. Díez, and M. V. Andrés, “Analog photonic fractional signal processing,” Prog. Optics, vol. 63, pp. 93-178, 2018.

[2] R. Slavík, Y. Park, M. Kulishov, R. Morandotti, and J. Azaña, “Ultrafast all-optical differentiators,” Opt. Express, vol. 14, pp. 10699-10707, Oct. 2006. 
[3] N. Q. Ngo, "Design of an optical temporal integrator based on a phase-shifted fiber Bragg grating in transmission,” Opt. Lett., vol. 32, pp. 3020-3022, Oct. 2007.

[4] M. A. Muriel, J. Azaña, and A. Carballar, "Real-time Fourier transformer based on fiber gratings," Opt. Lett., vol. 24, pp. 1-3, Jan. 1999.

[5] C. Cuadrado-Laborde and M. V. Andrés, "In-fiber all-optical fractional differentiator,” Opt. Lett., vol. 34, pp. 833-835, Mar. 2009.

[6] C. Cuadrado-Laborde and M. V. Andrés, "Proposal and design of an in-fiber all-optical fractional integrator,” Opt. Commun., vol. 283, pp. 5012-5015, Dec. 2010.

[7] L. Poveda-Wong, A. Carrascosa, C. Cuadrado-Laborde, J. L. Cruz, A. Díez, and M. V. Andrés, "Longperiod grating assisted fractional differentiation of highly chirped light pulses,” Opt. Commun., vol. 363, pp. 37-41, Mar. 2016.

[8] L. Poveda-Wong, A. Carrascosa, C. Cuadrado-Laborde, J. L. Cruz, and M. V. Andrés, "Experimental demonstration of fractional order differentiation using a long-period grating-based in-fiber modal interferometer,” Opt. Commun., vol. 380, pp. 35-40, Dec. 2016.

[9] C. Cuadrado-Laborde, A. Carrascosa, A. Díez, J. L. Cruz, and M. V. Andrés, "Photonic fractional Fourier transformer with a single dispersive device,” Opt. Express, vol. 21, pp. 8558-8563, Apr. 2013.

[10] L. Poveda-Wong, A. Carrascosa, C. Cuadrado-Laborde, J. L. Cruz, A. Díez, and M. V. Andrés, "Instantaneous frequency measurement by in-fiber 0.5th order fractional differentiation," Opt. Commun., vol. 371, pp. 89-92, Jul. 2016.

[11] C. Cuadrado-Laborde, A. Carrascosa, P. Pérez-Millán, A. Díez, J. L. Cruz, and M. V. Andrés, "Phase recovery by using optical fiber dispersion,” Opt. Lett., vol. 39, pp. 598-601, Feb. 2014.

[12] C. Cuadrado-Laborde, and M. V. Andrés, "Design of an ultra-broadband all-optical fractional differentiator with a long-period fiber grating,” Opt. Quant. Electron., vol. 42, pp. 571-576, Sept. 2011.

[13] L. Poveda-Wong, J. L. Cruz, M. Delgado-Pinar, X. Roselló-Mechó, A. Díez, and M. V. Andrés, "Fabrication of long period fiber gratings of subnanometric bandwidth," Opt. Lett., vol. 42, pp. 1265-1268, Apr. 2017. 